\title{
Mobilização Neural de Butler e Reiki como intervenção fisioterápica para capsulite adesiva crônica
}

\author{
Butler Neural Mobilization and Reiki as physiotherapy of \\ cronic adhesive capsulitis
}

\author{
Lillian Bondezan Holovatino ${ }^{1}$, Acary Souza Bulle Oliveira ${ }^{2}$, Sissy \\ Veloso Fontes ${ }^{3}$
}

\begin{abstract}
1.Fisioterapeuta, Especialização em Teorias e Técnicas para Cuidados Integrativos pela UNIFESP, Pósgraduanda em Gerontologia pelo Hospital Israelita Albert Einstein. São Paulo-SP, Brasil.

2.Médico Neurologista, Pós-doutor em Neurologia, Professor Afiliado do Departamento de Neurologia e Neurocirurgia da UNIFESP. São Paulo-SP, Brasil.

3.Fisioterapeuta, Psicóloga, Professora de Educação Física, Doutora em Ciências/Neurologia; Professora Afiliada do Departamento de Neurologia e Neurocirurgia da UNIFESP. São Paulo-SP, Brasil.
\end{abstract}

\begin{abstract}
Resumo
Introdução. Capsulite adesiva é uma síndrome multifatorial que inclui mudanças morfológicas e anatômicas da articulação do ombro acometido, seu diagnóstico é inicialmente clínico, marcado por significativa diminuição da amplitude de movimento e dor no ombro. Dentre os recursos da fisioterapia, a Mobilização Neural de Butler é uma técnica de mobilização manual, utilizada para melhora da amplitude de movimento e dor; o Reiki é uma prática de imposição de mãos, reconhecida como prática integrativa e complementar pelo Ministério da Saúde, devido intervir, por meio da energia vital nos aspectos físico, emocional, mental e espiritual. Objetivo. Investigar os efeitos de um programa fisioterápico utilizando a técnica de Mobilização Neural de Butler associada ao Reiki em estudo de caso de capsulite adesiva crônica. Método. Foram aplicadas 10 sessões de fisioterapia, utilizando da técnica de Mobilização Neural de Butler associada ao Reiki, e investigada a amplitude de movimento, força muscular e dor do ombro, funcionalidade ou desempenho nas atividades de vida diária, e variações de humor antes e imediatamente após o tratamento. Resultados. Houve melhora em todos os aspectos avaliados. Conclusão. A Mobilização Neural de Butler associada ao Reiki, como intervenção fisioterápica mostrou trazer benefícios ao tratamento de um paciente com capsulite adesiva crônica.
\end{abstract}

Unitermos. Bursite; Fisioterapia; Dor; Reiki; Práticas Integrativas e Complementares; Cuidados Integrativos

\begin{abstract}
Introduction. Adhesive capsulitis is a multifactorial syndrome that includes morphological and anatomical changes of the affected shoulder joint, its diagnosis is initially clinical, marked by significant decrease in range of motion and pain in the shoulder. Among the physiotherapy resources, Butler's Neural Mobilization is a manual mobilization technique, used to improve range of motion and pain; Reiki is a hands-on practice, recognized as an integrative and complementary practice by the Ministry of Health, due to intervene, through vital energy in the physical, emotional, mental and spiritual aspects. Objective. To investigate the effects of a physiotherapeutic program using the Butler Neural Mobilization technique associated with Reiki in a case study of chronic adhesive capsulitis. Method. Ten sessions of physiotherapy were applied, using Butler's Neural Mobilization technique associated with Reiki, and investigated the range of motion, muscle strength and shoulder pain, functionality or performance in daily life activities, and mood swings before and immediately after the treatment. Results. There was improvement in all aspects evaluated. Conclusion. Butler's Neural Mobilization associated with Reiki as a physiotherapeutic intervention has shown benefits in the treatment of a patient with chronic adhesive capsulitis.
\end{abstract}

Keywords. Bursitis; Physiotherapy; Pain; Reiki; Integrative and Complementary Practices; Integrative Care 


\section{INTRODUÇÃO}

Capsulite adesiva (CA) é uma síndrome multifatorial que inclui mudanças morfológicas e anatômicas da articulação glenoumeral; que causa dor, devido à compressão excessiva na articulação subacromial, diminuição da amplitude de movimento (ADM), fraqueza muscular, e, consequentemente alteração funcional do membro superior acometido ${ }^{1}$. Seu diagnóstico inicial é clinico, sendo que, os exames radiológicos só apresentarão alterações nos estágios mais avançados do quadro clínico ${ }^{1}$.

Estima-se que, hoje, no Brasil de 3 a $5 \%$ da população de adultos entre 40 e 60 anos sofram de CA, sendo essa proporção maior no sexo feminino (2:1), e, considerando a articulação do ombro, esta é a disfunção neuromuscular mais frequente ${ }^{2,3}$.

Por ser uma síndrome que acomete diferentes estruturas, como ossos, cartilagem, ligamentos e tendões, a capsulite adesiva apresenta melhora significativa quando submetida à fisioterapia, dentre suas diferentes técnicas ${ }^{4}$. Dentre elas, a Mobilização Neural de Butler (MN) é considerada uma técnica de mobilização manual e tem como base teórica o deslizamento do tecido nervoso sob a fáscia muscular, quando do manuseio do seguimento corporal em questão, sendo assim, vale lembrar que o 
sistema nervoso (SN) apresenta uma característica importante e notável, sua mobilidade pode agir dependente ou independente dos sistemas adjacentes, sendo assim, ele consegue adaptar-se ao movimento de duas formas distintas: pelo alongamento muscular e pela mobilização das articulações ${ }^{5-7}$.

A MN quando comparada ao alongamento muscular convencional apresenta melhores resultados em relação à ADM, força muscular e diminuição da dor, isso ocorre, principalmente devido a forma como o movimento da técnica é realizado, respeitando, principalmente os sintomas locais, favorecendo a liberação das aderências perineurais locais ou sistêmicas ${ }^{8}$.

Outros procedimentos fisioterápicos para dor e disfunção são as técnicas alternativas e complementares ${ }^{6}$; esses, atualmente são conhecidas como práticas integrativas e complementares (PICS), descritas nas portarias de no 971/GM/MS em 03 de Maio de 2006 e de no 145/2017 em 13 de Janeiro de 2017, da Política Nacional de Práticas Integrativas e Complementares (PNPIC), reconhecidas pelo Ministério da Saúde para o Sistema Único de Saúde (SUS). Dentre eles, está incluso a técnica de imposição de mãos denominada Reiki ${ }^{6}$.

Reiki é uma palavra de origem japonesa, composta por dois termos: "Rei" Universal e "Ki" Energia Vital, sendo um método científico de cuidado, reconhecido como prática complementar pela Organização Mundial de Saúde (OMS). Apesar de ser uma técnica que envolve "iniciação" em sua 
formação, não é relacionada a nenhuma religião, tornandose acessível a qualquer um que queira aprender sobre ela ${ }^{9}$. Por envolver o corpo físico, emocional, mental e espiritual, o Reiki é uma técnica de imposição de mãos muito utilizada e, é capaz de despertar em seus praticantes equilíbrio mental e espiritual, promovendo relaxamento global e bem estar $^{9-11}$.

Sendo assim, o objetivo desse estudo foi investigar os efeitos de um programa fisioterápico utilizando a técnica de Mobilização Neural de Butler associada ao Reiki em um estudo de caso de capsulite adesiva crônica, em relação a flexibilidade articular, força muscular, desempenho nas atividades de vida diária, variações do humor, funcionalidade (capacidades e incapacidades) e dor.

\section{MÉTODO}

Trata-se de um estudo de caso: mulher de 52 anos, solteira, massagista, residente em São Paulo, com o diagnóstico de capsulite adesiva estágio III em ombro esquerdo, desde o final do $2^{\circ}$. semestre de 2015 ; e, quadro de mialgia em ombro direito, no início de 2017. Foram utilizadas para avaliação: anamnese, avaliação da amplitude de movimento (ADM) por meio da goniometria, força muscular, e investigação quanti e qualitativa da dor (intensidade, localização, duração, aspectos relacionados ao alivio e piora da dor). Em adição, inclui-se a investigação do estado de humor com base na Escala de Likert; e 
investigou-se sobre as incapacidades funcionais utilizandose do Índice de dor e incapacidade no ombro - Brasil: Shoulder Pain and Disability Index (SPADI) versão brasileira $^{12}$.

A avaliação foi realizada antes de iniciar o programa fisioterápico e imediatamente após o término do mesmo, ou seja, após quatro meses de intervenção.

O Programa contou com 10 sessões, e foi elaborado e conduzido pela mesma fisioterapeuta (autora desse estudo), com base em revisão da literatura e experiência clínica da autora do estudo, divididas em uma sessão por semana ao longo de quatro meses, sendo uma sessão semanal com duração de aproximadamente 50 minutos, com três semanas de pausa entre a quinta e a sexta sessão, por conta de uma viagem previamente marcada pela paciente, participante do estudo.

Cada sessão contou, inicialmente com a Mobilização Neural de Butler, utilizando de movimentos oscilatórios por 40 segundos dos seguintes testes de tensão7: iniciando com o teste de Flexão Cervical Passiva (PNF), Flexão Cervical Passiva associada a Flexão torácica, Extensão Cervical Passiva (PNE), Teste de Tensão do Membro Superior 1 e 2 (ULTT1 e ULTT2), e Elevação da Perna Estendida (SLR).

Após a aplicação da mobilização neural, foram realizadas aplicações de Reiki por 3 minutos em cada região corporal, quais sejam: topo do crânio, olhos, orelhas, garganta, ombros (no local em que o indivíduo referiu 
maior dor), região abaixo do processo xifoide (plexo solar); região do externo com região abaixo do processo xifoide (coração - integração plexo solar) e, planta dos pés. Para controle de tempo da fisioterapeuta foi colocada uma música com sons da natureza com um sino a cada três minutos, a música utilizada foi a mesma em todas as sessões.

\section{RESULTADOS}

Observou-se melhora da amplitude de movimento (ADM) do ombro, bilateralmente. No ombro esquerdo, o movimento de flexão, na avaliação inicial apresentava $56 \%$ da ADM total possível, enquanto que na reavaliação passou a apresentar $76 \%$ da máxima amplitude, ganhando no período de tratamento 20,6 pontos percentuais; a extensão apresentou $82 \%$ do total na avaliação inicial e, $96 \%$ do máximo na reavaliação, melhora de 13,3 pontos percentuais; a abdução passou de $50 \%$ na primeira avaliação para $84 \%, 34,4$ pontos percentuais, a adução passou de $90 \%$ para $100 \%, 10$ pontos percentuais de melhora, rotação lateral passou de $89 \%$ a $98 \%$ da máxima, melhora de 8,9 pontos percentuais e por fim, a rotação medial obteve melhora de 17,8 pontos percentuais, passando de $69 \%$ para $87 \%$. O membro superior direito (MSD) apresentou melhora naquelas que não apresentavam o total da amplitude de movimento na avaliação inicial, sendo flexão que passou de $89 \%$ para 
97\% do possível, 7,8 pontos percentuais de melhora, extensão que apresentava $87 \%$ do total e apresentou melhora de 13,3 pontos percentuais passando para $100 \%$ e abdução que passou de $93 \%$ na primeira avaliação para $100 \%$, apresentando 6,7 pontos percentuais, no MSD os movimentos adução, rotação lateral e medial já apresentavam $100 \%$ na avaliação inicial apresentaram o mesmo resultado na reavaliação.

A força muscular em membro superior esquerdo (MSE) apresentou melhora de 3 para 4 pontos na avaliação clínica em abdução, rotação lateral e extensão, enquanto que as demais (adução, rotação medial e flexão) não apresentaram diferença, observando que em abdução tanto em MSE quanto em MSD ela referiu dor na avaliação inicial e não voltou a referir na reavaliação. Em MSD todos os grupos musculares obtiveram pelo menos 4 na avaliação e apresentou melhora em rotação medial (de 4 para 5 pontos).

A avaliação de humor por meio da escala Likert apresentou melhora logo após a primeira sessão, passando de 1,8 na média para 2,8 após o período apresentado, também pode-se observar que após a primeira avaliação a pontuação máxima passou de 3 (amena) para 4 (feliz ou animada).

As atividades de vida diária, tal como as competências funcionais do ombro apresentaram melhora significativa, as quais puderem ser observadas a partir do questionário Shoulder pain disability index validado para o português 
como SPADI - Brasil. Todos os itens avaliados obtiveram melhora.

O escore de incapacidade de MSE passou de 80 pontos para 11,24 e MSD passou de 88 para 8 pontos na reavaliação. O escore de dor passou de 61,25 para 6,25 pontos em MSE e de 72 para 38 pontos em MSD.

\section{DISCUSSÃO}

Em relação à melhora da ADM, o estudo de Santos e Domingues $(2008)^{13}$ apresentaram resultados, também satisfatórios em relação à ADM, quando da utilização da MN em membros inferiores. Como o SN é responsável não só por conduzir o impulso nervoso, mas, adaptar-se mecanicamente ao estado dos músculos e articulações, quando mobilizados produz um movimento neurodinâmico local e sistêmico que, por consequência oferece melhora fisiológica nas estruturas próximas a ele.

Além disso, a mobilização neural (MN) agindo por meio de movimentos oscilatórios e menos estressantes para o sistema musculoesquelético apresenta resultados melhores se comparados ao alongamento muscular clássico; Machado e Bigolin (2010), que realizaram um estudo comparando tais técnicas, atribuem a eficácia da MN na flexibilidade muscular à restauração do movimento por meio da elasticidade do $S N$, promovendo retorno às funções normais do músculo. Resultado que pode ser atribuído, 
também a diminuição da dor e de espasmos musculares favorecidos pela Mobilização Neural de Butler ${ }^{14}$.

Quanto à força muscular obtivemos melhora nos grupos musculares que não apresentavam a melhor pontuação possível, no momento da primeira avaliação, corroborando aos resultados do estudo de Machado e Bigolin $(2010)^{14}$ que, ao avaliar a força muscular de preensão palmar, utilizando os testes ULTT 1, 2 e 3 da MN, verificaram aumento da mesma. Lopes et al $(2010)^{15}$ ao comparar os resultados em relação à melhora da força muscular de quadríceps de intervenções fisioterapêuticas com alongamento muscular convencional e com MN, concluíram que a $\mathrm{MN}$ promoveu melhora mais significativa no desempenho muscular, pois, facilita o movimento do fluxo axoplasmático, melhorando a irrigação e a qualidade do tecido muscular inervado pela estrutura mobilizada, potencializando a ativação e desempenho do fuso neuromuscular ${ }^{14,15}$.

As melhoras encontradas no questionário SPADI Brasil estão diretamente relacionados com a melhora da ADM e da força muscular, pois, eles contribuem para melhor realização das tarefas funcionais. Machado e Bigolin $(2010)^{14}$ destacam que a flexibilidade muscular tem ação direta na realização das atividades de vida diária, pois, quando realizadas com maior amplitude de movimento, podem ganhar força, rapidez e fluidez, o que favorece tais atividades e, por sua vez, diminuem a prevalência de lesões ${ }^{14}$. 
O aspecto dor é o aspecto mais complexo avaliado, pois, ele leva em conta uma questão subjetiva entendida de forma diferente para cada indivíduo. Por este motivo, o questionário SPADI - Brasil teve um papel importante na comparação da dor no início e ao término da realização do programa.

O Reiki tem uma função importante na promoção do relaxamento e diminuição do estresse, aspectos que associados levam ao relaxamento muscular, e, consequentemente ao alivio da dor, ele também pode facilitar as liberações emocionais, gerando um efeito visível e imediato ao tratamento ${ }^{16}$.

\section{CONCLUSÃO}

Pode-se inferir que a intervenção fisioterapêutica que utiliza da Mobilização Neural de Butler associada ao Reiki é satisfatória no tratamento de capsulite adesiva crônica em relação à melhora da amplitude de movimento, força muscular, estado de humor e capacidade funcional; além da diminuição da dor.

\section{REFERÊNCIAS}

1.Ejnismann B, Monteiro GC, Uyeda LF. Ombro doloroso. Einstein 2008;6(suppl 1):133-7.

2.Ferreira Filho AA. Capsulite adesiva. Rev Bras Ortop 2005;40:56574.

3.Cohen M, Amaral MV, Brandão BL, Pereira MR, Monteiro M, Filho GRM. Avaliação dos resultados do tratamento cirúrgico artroscópico da capsulite adesiva. Rev Bras Ortop 2013;48:272-7.

http://dx.doi.org/10.1016/j.rbo.2012.08.004 
4.Georgiannos D, Markopoulos G, Devetzi E, Bisbinas I. Adhesive capsulitis of the shoulder. Is there consensus regarding the treatment? A comprehensive Review. Open Orthop J 2017;11:65-75. http://dx.doi.org/10.2174/1874325001711010065

5.Cavalcante CCL, Rodrigues ARS, Dadalto TV, Silva EB. Evolução científica da fisioterapia em 40 anos de profissão. Fisioter Mov 2011;24:513-22. $\quad$ http://dx.doi.org/10.1590/S0103$\underline{51502011000300016}$

6. Fontes SV, Alves MAF, Ottoboni C. Classificação fisioterapêutica. In: Fontes SV, Fukujima MM, Cardeal JO. Fisioterapia neurofuncional Fundamentos para a prática, São Paulo: Atheneu, 2007.

7.Butler DS. Mobilização do sistema nervoso. Barueri: Manole; 2003.

8.Lima MO, Vasconcelos TB, Arcanjo GN, Soares RJ. A eficiência da mobilização neural na reabilitação da lombalgia: uma revisão de literatura. Rev Bras Cien Saúde 2012;10:45-9. https://doi.org/10.13037/rbcs.vol10n31.1390

9. Costeira CRB. A influência da terapia de Reiki em indicadores de saúde. Inter J Develop Educ Psychol 2009;1:465-72.

10.Almeida LF. Efeitos da técnica reiki nas dimensões biopsicossocioespiritual do ser humano: revisão e atualização da literatura. São Paulo: Universidade Federal de São Paulo; 2012.

11.Freitag VL, Dalmolin IS, Badke MR, Andrade A. Benefits of reiki in older individuals with chronic pain. Texto Contexto Enferm 2014;23:1032-40. 07072014001850013

http://dx.doi.org/10.1590/0104-

12.Martins J, Napoles BV, Hoffman CB, Oliveira AS. Versão brasileira do Shoulder Pain and Disability Index: Tradução, adaptação cultural e confiabilidade. Rev Bras Fisioter 2010;14:527-36.

http://dx.doi.org/10.1590/S1413-35552010000600012

13.Santos CF, Domingues CA. Avaliação pré e pós-mobilização neural para ganho de ADM em flexão do quadril por meio do alongamento dos isquiotibiais. ConScientiae Saúde 2008;7:48795. https://doi.org/10.5585/conssaude.v7i4.1389

14. Machado GF, Bigolin SE. Estudo comparativo de casos entre a mobilização neural e um programa de alongamento muscular em lombálgicos crônicos. Fisioter Mov 2010;23:545-54. http://dx.doi.org/10.1590/S0103-51502010000400005

15.Lopes RSD, Barja PR, Matos LKBL, Delmondes FF, Lopes PFD, Silva KAS, et al. Influência do alongamento muscular e da mobilização neural sobre a força do músculo quadríceps. ConScientiae Saúde 2010;9:6039. https://doi.org/10.5585/conssaude.v9i4.2355

16.Sousa M. A psicossomática e o Reiki. Psicologia PT 2012;1-14. 